\title{
An Insight into Torque Production and Power Factor in Transverse-Flux Machines
}

\author{
J. R. Anglada, Student Member, IEEE and S. M. Sharkh, Senior Member, IEEE
}

\begin{abstract}
Despite transverse flux machines (TFMs) being intrinsically three-dimensional, it is still possible to model them analytically using relatively simple models. This paper aims to provide an insight into the behaviour of TFMs using a compact equation, which relates torque to the electric and magnetic loadings of the machine and a flux factor. The flux factor is also used to estimate the flux linkage and therefore the power factor of this kind of machines. It is shown that the low power factor of TFMs is not only due to leakage but also due to the nature of the electromagnetic interaction that takes place. The TFM developed at the University of Southampton is used as the basis of a case study to illustrate the trade-off between torque density and power factor, and to provide some design guidelines. The analytical results are verified using finite elements analysis and experimental data.
\end{abstract}

Index Terms-Analytical models, permanent magnet machines, power factor, torque, transverse-flux machines.

\section{INTRODUCTION}

$\mathbf{T}$ HE massive development of renewable energy sources, such as wind and tidal, has generated a great interest in high torque density electric generators. For example, the typical rotation speed of wind turbines is between 5 and $25 \mathrm{rpm}$ which necessitate the installation of a gearbox when traditional generators are used, making the system less efficient and reliable [1]-[3]. Direct drive high torque density electric generators, such as transverse flux machines (TFMs), have therefore been of interest as potential alternatives. But these machines tend to have an inherently low power factor [4]; which has hindered their acceptance. But as will be shown later in this paper, the power factor and torque density are interlinked and increasing one reduces the other. This can be the key to unlocking the potential of these machines through a happy compromise between torque density and power factor.

TFMs are inherently three-dimensional devices that require 3D FEA, which has been the main tool used for the analysis and design of many machines reported in the literature [3], [5]-[9]. By contrast there are significantly fewer papers on analytical methods [10], [11]. In comparison, analytical methods are less accurate than FEA but they are much faster and provide a greater insight.

This work was supported by EPSRC and by TSL Technology.

J. R. Anglada and S. M. Sharkh are with the Mechatronics Research Group, Engineering Sciences, Faculty of Engineering and the Environment, University of Southampton, Highfield Campus, Southampton SO17 1BJ, United Kingdom (e-mail: j.renedo-anglada@soton.ac.uk; suleiman@soton.ac.uk).
In this paper we develop the analytical theory described in [10] further to derive expressions for torque and power factor of the TFM described in [10], [11], which is also referred as variable-reluctance permanent-magnet (VRPM) machine. The methodology can be extended to other TFM topologies.

The insight gained from the torque and power factor expressions, which are validated using FEA and experimental data, is used to develop design guidelines for the selection of the key parameters of these machines.

Section II describes single-sided TFM that was developed at the University of Southampton [10], [11]. Next, in section III we describe the background theory of torque production and the insight that can be obtained from it. Section IV studies the calculation of the power factor, the reasons for the low values in TFMs and its relationship with the torque equation. In section $\mathrm{V}$, the equation for torque and power factor is applied to the machine under study and the results are validated by FEA and experimental results. The strong relationship between the power factor and torque density is also illustrated. The insight gained from the case study is used to develop design guidelines. Conclusions are presented in section VI.

\section{The SINGLE-Sided TFM UNDER STUdY}

Fig. 1 shows the front view and the cross-section of the machine under study, which is an inverted surface-magnet configuration with an outer rotor. The stator is two-phase, each phase has a circular coil that links and magnetizes $20 \mathrm{C}$ cores producing a homopolar magnetic field distribution. The radial flux interacts with an array of 40 permanent magnets with alternating polarity. The dimensions of the machine studied are shown in table I.

TABLE I

PARAMETERS OF THE TFM

\begin{tabular}{lcc}
\hline Quantity & Symbol & Value \\
\hline Stator radius & $R_{s}$ & $73 \mathrm{~mm}$ \\
Rotor radius & $R_{r}$ & $78.5 \mathrm{~mm}$ \\
Air-gap length & $g$ & $5.5 \mathrm{~mm}$ \\
Magnet thickness & $d_{m}$ & $4.51 \mathrm{~mm}$ \\
Pole pitch & $\theta_{\lambda}$ & $18^{\circ}$ \\
Tooth pitch & $\theta_{t}$ & $7.02^{\circ}$ \\
Slot pitch & $\theta_{s}$ & $10.98^{\circ}$ \\
Number of C-cores & $N_{c}$ & 20 \\
Number of turns & $N_{w}$ & 230 \\
Number of phases & $q$ & 2 \\
\hline
\end{tabular}

The C-cores are made of laminated steel and they are aligned in radial planes as it is shown in Fig. 1. The rotor is 


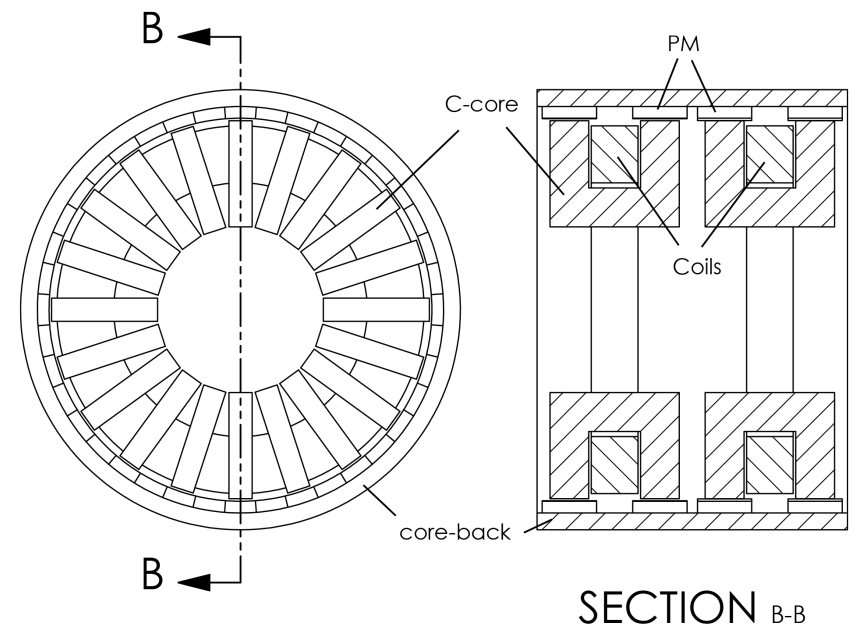

Fig. 1. Front view and cut of a single sided TFM under study.

a solid drum, which forms a magnetic yoke for the flux of the neodymium PMs. Each phase has two arrays of alternating polarity PMs, which are placed in such a way that in the aligned position with the $\mathrm{C}$-cores they tend to produce a flux in the same direction. Fig. 2 shows a quarter of a 3D FEA model of the TFM under study; the arrows represent the fluxdensity due to the PMs in the aligned position.

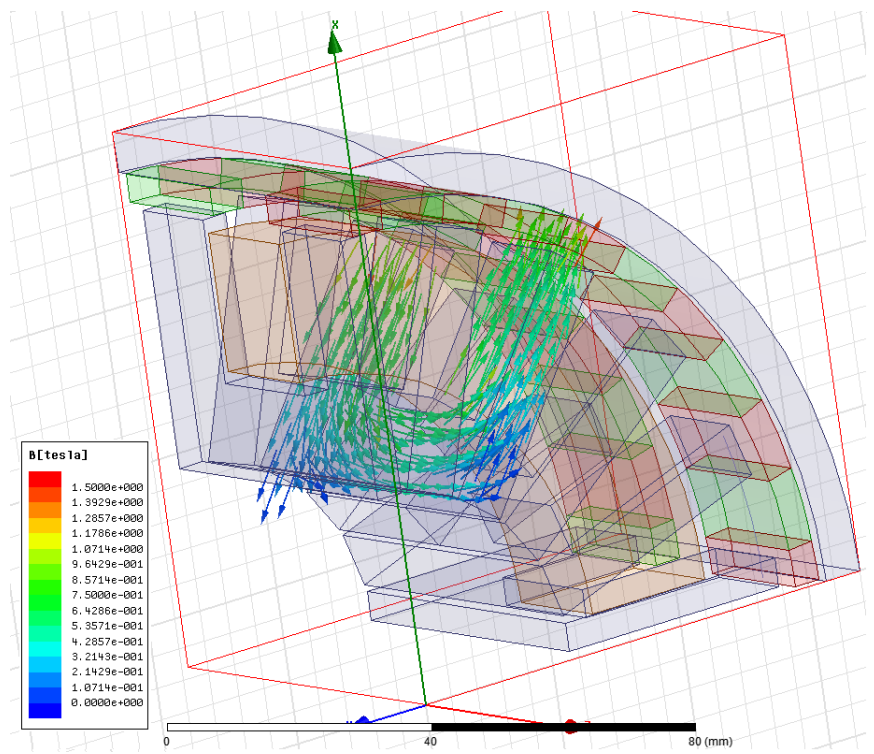

Fig. 2. Flux through the C-core due to the PMs.

\section{THEORY OF TORQUe PRODUCTION}

The methodology presented here is based on the work done by Harris et al. [10], [11]. If the PMs are replaced with equivalent current sheets we can apply the BiL principle to calculate torque, but now $i$ is the equivalent current of the magnets and $B$ is the magnetic field produced by the stator windings. Fig. 3 shows the developed model of the air-gap of the TFM with the equivalent currents shown as dots and crosses (shown only for one PM).

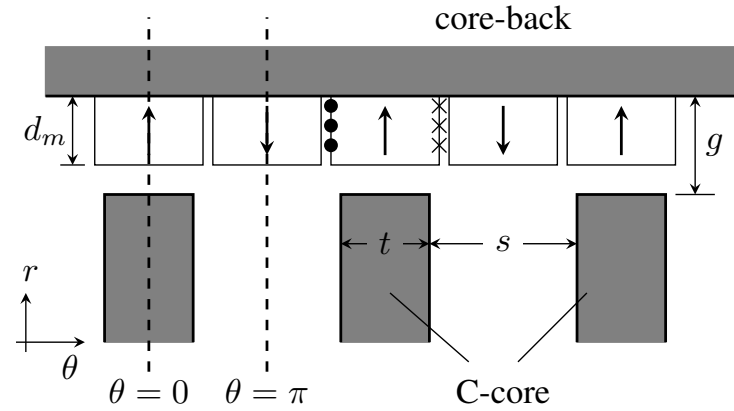

Fig. 3. Developed model of the TFM.

Let us assume a square-wave current waveform for the following deduction of the torque equation. In half a period the positive equivalent current sheets of the PMs move through the top of the teeth where the flux density is strong (from $\theta=-\pi / 2$ to $\theta=\pi / 2$ ) and the negative equivalent current sheet moves through the slot region where the flux density is weak (from $\theta=\pi / 2$ to $\theta=3 \pi / 2)^{1}$. The net force that is effectively helping to produce torque is the force on the positive current sheets minus the force on the negative current sheets. Fig. 4 shows a simplified drawing of the air-gap of the TFM with the positive and negative currents at a distance $\delta$ of the core-back. In reality the force is distributed all along the magnet's equivalent current sheets. However, for the following analysis the equivalent current sheets are represented as current points that take into account the average force along the edges of the PMs without losing any generality.

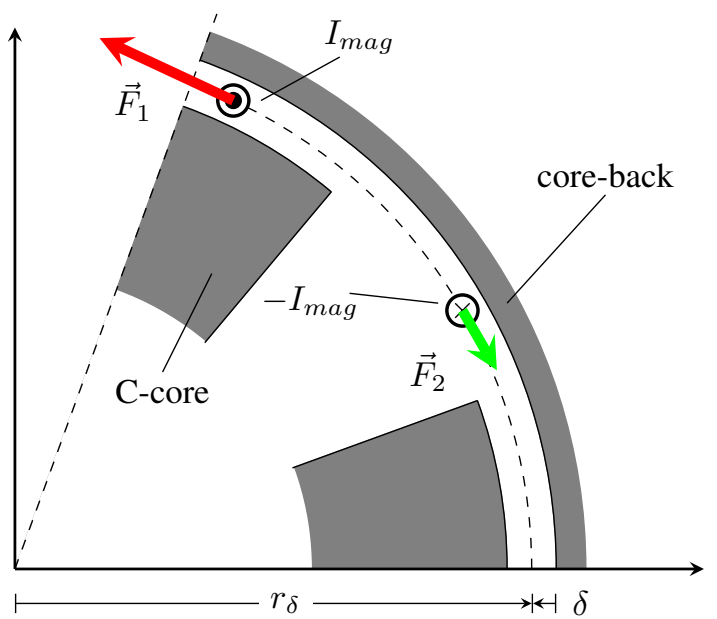

Fig. 4. Forces applied to the equivalent current loop at a distance $\delta$.

Based on this, we can simply calculate the average positive torque per phase as

$$
T=2 q N_{c} K_{B} B_{s} I_{m a g} L_{e q} R,
$$

where $K_{B}$ is the flux factor [10], [11], $I_{\text {mag }}$ the equivalent

\footnotetext{
${ }^{1}$ At this instant of time the current changes from positive to negative and the same process repeats with the PMs of the inverse polarity.
} 
current of the PMs, $L_{e q}$ the axial length, $R$ the effective radius and $B_{s}$ is the radial flux-density produced by the stator's MMF as defined by Harris et al. [10]:

$$
B_{s}=\frac{\mu_{0} F}{g} .
$$

where $F$ is the stators MMF from one $\mathrm{C}$-core head to the core-back calculated as

$$
F=\frac{1}{2} N_{w} I
$$

The value of the equivalent current of the PMs is obtained as follows

$$
I_{m a g}=2 \mathcal{M} \cdot d_{m}=2 F_{m},
$$

where $\mathcal{M}$ is the magnetisation and $d_{m}$ the magnet thickness.

The flux factor $K_{B}$ accounts for the fact that part of the flux is not producing torque; in fact it is doing the opposite; it is reducing the net force and therefore reducing the total torque. Let us proceed to deduce the expression of $K_{B}$.

The radial component of the stators magnetic field distribution in the air-gap, which is the component that effectively produces torque, can be expressed as a Fourier cosine series [12]-[14]. For our case this is

$$
B_{r}(\theta)=B_{s} \bar{\lambda}_{r}\left[1+\sum_{n=1}^{\infty} \gamma_{n} \cos (n \theta)\right],
$$

where $\bar{\lambda}_{r}$ and $\gamma_{n}$ are coefficients that need to be determined considering that they represent the average value along the magnet thickness. Fig. 5 shows the waveform of the radial component of the magnetic field in blue. The area under the curve is the total magnetic flux; the red represents the useful flux associated with the positive force and the green one the useless magnetic flux associated with the negative force.

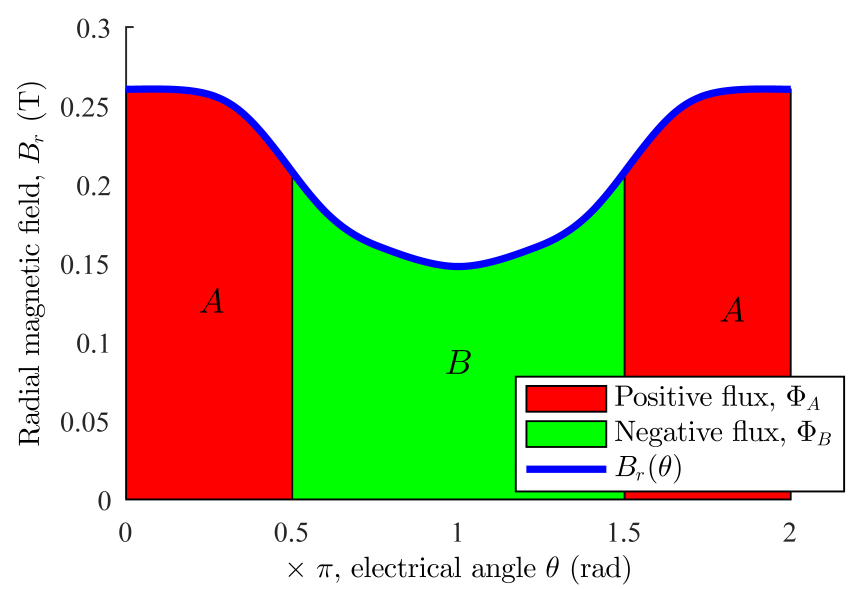

Fig. 5. Waveform of the radial component of the stator's magnetic field distribution and the corresponding useful/useless flux.

Let us call $\Phi_{A}$ the positive flux, red in Fig. 5, and $\Phi_{B}$ the negative flux, green in Fig. 5. Then, the net force is proportional to the difference $\Phi_{A}-\Phi_{B}$. Accordingly, the flux factor accounts for the average value of the force normalised, in this case as

$$
K_{B}=\frac{1}{B_{s}} \frac{\Phi_{A}-\Phi_{B}}{\pi} .
$$

Operating with the expression of the magnetic field distribution (5) it can be shown that

$$
K_{B}=\frac{4}{\pi} \bar{\lambda}_{r}\left[\gamma_{1}-\frac{\gamma_{3}}{3}+\frac{\gamma_{5}}{5}-\ldots\right] .
$$

This expression of the flux factor is consistent with references [10], [11], [14]. The TFM described in section II has a flux factor

$$
K_{B} \approx 0.3125,
$$

which is consistent with the torque obtained using FEA shown in [11], [14].

The previous deduction was implicitly based on the assumption of a square wave current. This machine is meant to operate with a sinusoidal waveform. In this condition the value of $B_{s}$ is not constant during an electrical cycle because the current is changing. Therefore,

$$
B_{s}(\theta)=\frac{\mu_{0} F(\theta)}{g}=\frac{\mu_{0} \hat{F} \cos (\theta)}{g},
$$

where $\hat{F}$ is

$$
\hat{F}=\frac{1}{2} \sqrt{2} N_{w} I_{R M S} \cdot
$$

It can be shown that the expression of flux factor calculated analogously as in the previous case but considering sinusoidal current is

$$
K_{B}=\bar{\lambda}_{r} \gamma_{1}
$$

It is important to point out the significance of Fig. 5. The torque is proportional to red area minus the green area, which means that all the magnetic flux $\Phi_{B}$ is producing a negative torque. The flux factor captures this effect that is inherent to the homopolar field distribution of this TFM.

\section{Relationship with the Power Factor}

The reasons for the low power factor of TFMs are analysed in detail in [4]. The conclusions are that besides the leakage, which is high in these machines, the main cause is the ineffective use of magnetic flux $\left(\Phi_{B}\right.$ is producing a negative torque). However, still many publications state that the low power factor is due to leakage in the classical way [1], [15]-[18]. Some authors call leakage to the flux produced by the PMs that are not aligned with the C-cores, which diminishes the total flux. Still, this flux is not leakage in the classical way because it is crossing the air-gap but in the opposite direction [19]-[21]. The aim of this section is to show the relationship between the flux factor (and therefore the torque) and the power factor.

To operate in maximum torque condition the current, $I$, has to be in phase with the back EMF, $E$; so this means that current only has q-axis component $I_{q}$. Considering that there 
is not saliency in this machine $X_{d}=X_{q}=X$. The phasor diagram is shown in Fig. 6.

The reactance is due to the self-inductance of the coils, it can be separated into two different terms one due to the flux that crosses the air-gap, $L_{g}$, and one due to the leakage in the axial direction, $L_{l}$.

The value of $L_{g}$ can be estimated using the expression of the magnetic field distribution in the air-gap to obtain the flux. For a given current $i$ the inductance is calculated as follows

$$
L_{g}=N_{c} \frac{N_{w} \Phi_{\text {core }}}{i},
$$

where $\Phi_{\text {core }}$ is the total flux passing through one C-core calculated as follows

$$
\Phi_{\text {core }}=\hat{B} \bar{\lambda}_{r} L_{e q} \frac{2 \pi R}{N_{c}},
$$

with

$$
\hat{B}=\frac{\mu_{0} N_{w} i}{2 g},
$$

therefore the final expression of the inductance is

$$
L_{g}=\mu_{0} N_{w}^{2} L_{e q} \frac{\pi R \bar{\lambda}_{r}}{g},
$$

The leakage flux in the axial direction can be estimated using the expression for the slot leakage according to Say [19]:

$$
L_{l}=\mu_{0} N_{c} N_{w}^{2} \ell_{\text {core }}\left(\frac{h_{1}}{3 w_{s}}+\frac{h_{2}}{w_{s}}\right),
$$

where $h_{1}$ is the coil depth, $h_{2}$ is the difference between the slot depth and the coil depth, $w_{s}$ the slot width and $\ell_{\text {core }}$ the $\mathrm{C}$-core thickness. In this case the slot is the $\mathrm{C}$-core looked from the radial direction. The total reactance $X$ is calculated as follows

$$
X=\omega\left(L_{g}+L_{l}\right) .
$$

Normally the value of $X$ is much greater than the value of the resistance of the windings $R_{w}$. Therefore, the angle $\phi$ in Fig. 6 can be approximated as

$$
\phi \approx \tan ^{-1}\left(\frac{I_{q} X}{E}\right)
$$

To estimate the value of the back EMF, $E$, the principle of conservation of energy can be used. When operating in steady state conditions, at constant mechanical speed $\Omega$, the electromagnetic power in the shaft has to be the same as the electrical power if losses in the energy conversion process are ignored. The average power in the shaft can be easily obtained with the torque equation as follows

$$
P=q \cdot T \cdot \Omega=2 q N_{c} K_{B} B_{s} I_{m a g} L_{e q} R \cdot \frac{\omega}{N_{c}}
$$

On the other hand, if $I_{R M S}$ is the value of the fundamental harmonic of the current and $E_{R M S}$ is the value of the fundamental harmonic of the voltage. Then, the average electrical power is simply

$$
P=q \cdot I_{R M S} \cdot E_{R M S} .
$$

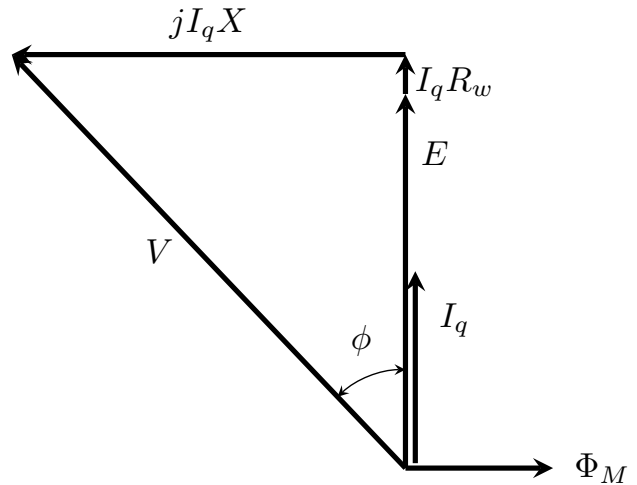

Fig. 6. Phasor diagram with current only in the quadrature axis.

Ignoring the power losses in the electro-mechanical energy conversion process, the electromagnetic power in the shaft is equal to the electrical power. Therefore, we can have a rough estimation of the amplitude of the fundamental of the back EMF as follows

$$
E_{R M S} \approx \frac{P}{q I_{R M S}}=\frac{2 K_{B} B_{s} I_{m a g} L_{e q} R \cdot \omega}{I_{R M S}},
$$

since $B_{s}$ depends on $I_{R M S}$, considering a sinusoidal current, as follows

$$
B_{s}=\frac{\mu_{0} F}{g}=\frac{\sqrt{2}}{2} \frac{\mu_{0} N_{w} I_{R M S}}{g} .
$$

Accordingly, the value of the back EMF is

$$
E_{R M S} \approx \frac{\sqrt{2} \mu_{0} \pi}{2} K_{B} F_{m} \omega N_{w} \frac{L_{e q} R}{g} .
$$

To better understand the expression let us define the value of the magnetic field in the air-gap produced by the PMs as

$$
B_{P M}=\frac{\mu_{0} F_{m}}{g}
$$

therefore, the expected flux linkage with the winding considering that only half of the magnets are producing flux (homopolar nature)

$$
\Phi_{P M}=\pi R L_{e q} B_{P M} N_{w},
$$

Combining the previous expressions, the RMS value of the back EMF is

$$
E_{R M S} \approx \frac{1}{\sqrt{2}} K_{B} \Phi_{P M} \omega
$$

which can be rewritten as

$$
E_{R M S} \approx k_{E} \omega
$$

where $k_{E}$ is the back EMF constant [22]. This expression has the same shape as the well-known formula that uses the EMF constant $k_{E}$ [22]. However, in this case the flux factor is multiplying the total flux because of this ineffective use of the $M M F$ that was described previously. Equation (26) illustrates very clearly the relationship of the EMF and torque since in this case both are proportional to the flux factor $K_{B}$. 


\section{Case Study}

This section shows how to choose the optimum number of C-cores for the topology of the TFM under study. If the objective is to maximise torque then we have to use (1) with $K_{B}$ calculated using (7). The magnetic field distribution in the air-gap was calculated using the complex permeance function [23], therefore we are assuming that there is no saturation. The effect of slotting was taken into account using the Schwarz-Christoffel Toolbox for Matlab [24].

Fig. 7 shows the average torque per phase versus the current obtained with the torque equation described in section III, the value measured in the lab and the results from 3D FEA. It can be appreciated that the methodology presented is accurate for unsaturated load conditions.

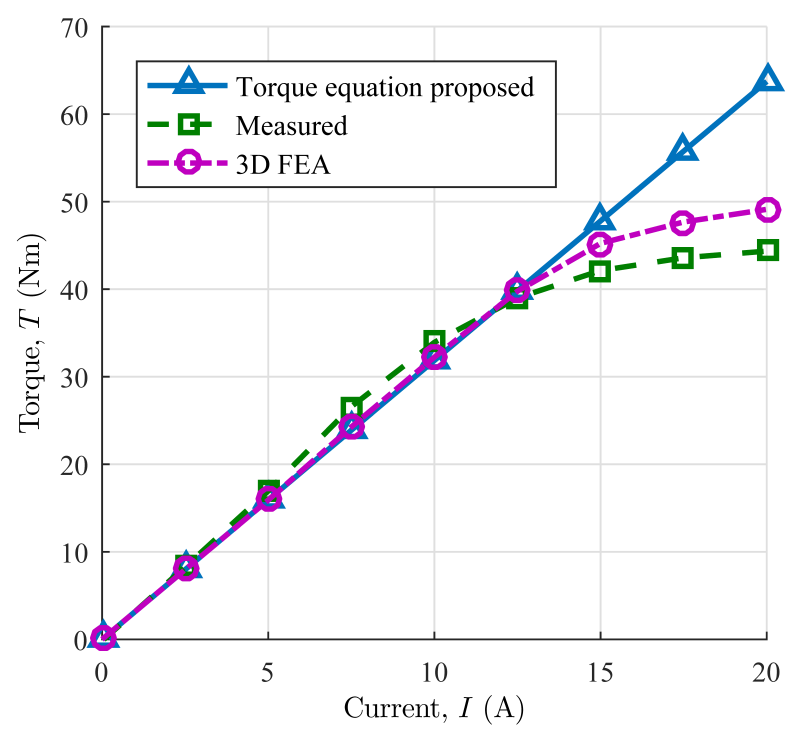

Fig. 7. Average torque per phase, machine's data from table I. Rated current is $10 \mathrm{~A}$.

For this example let us assume that the magnet thickness $d_{m}$, the air-gap length $g$ and the tooth-pitch ratio $t / \lambda$ remain constant [11]; $\lambda$ is the pole-pitch

$$
\lambda=\frac{2 \pi R}{N_{c}} .
$$

Under these conditions we can vary the number of Ccores, $N_{c}$, to study the performance. The magnet thickness affects in the design but the dominant effect in this case is the number of C-cores, therefore for this example the magnet thickness is assumed to be constant. In this case the torque is calculated using (1). Also, since we deduced an approximated expression to estimate the power factor it can be also calculated as a function of the number of C-cores.

Fig. 8 shows the average torque per phase (scale on the left hand side) together with the power factor and the flux factor (scale on the right hand side). Starting from $N_{c}=1 \mathrm{C}$ core as $N_{c}$ increases the average torque also increases until it reaches a maximum, which corresponds to $N_{c}=24 \mathrm{C}$-cores. However, the flux factor and the power factor are strictly

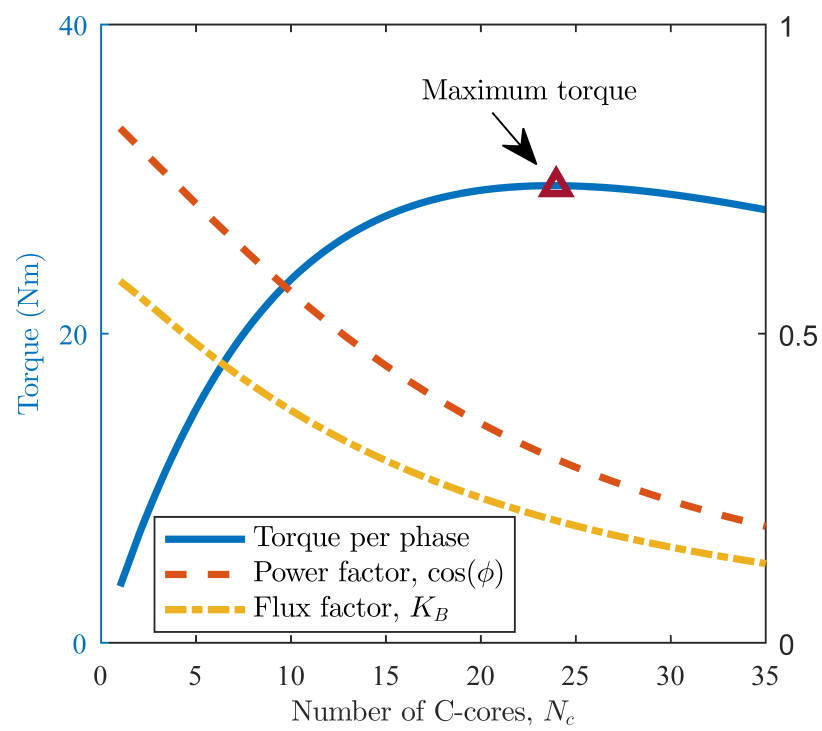

Fig. 8. Average torque per phase and power factor as a function of the number of C-cores.

decreasing. In fact the maximum torque is reached when the improvement of torque produced by an additional C-core is compensated by the penalty of a lower flux factor. Therefore, the optimum number of $\mathrm{C}$-cores does not correspond to the maximum value of the flux factor. This result was expected and is consistent with the work presented in [10], [11].

Now focusing on the power factor, we see very clearly the reasons of the poor power factor of machines that were designed only by maximising torque. Near $N_{c}=20$ C-cores the increase of torque per extra additional C-core is low but the decrease of the power factor is significant. For example, if we compare $N_{c}=15$ and $N_{c}=24$ C-cores the second case has $2.27 \mathrm{Nm}$ more torque. However, the power factor drops from 0.448 to 0.296 at full load. This lower power factor means that the power electronic converter has a higher power rating.

When designing a machine for a specific torque requirement (in this case let us say it is $32 \mathrm{Nm}$ ). Then, it is reasonable to ask: is it better to choose the number of $C$ cores to maximise torque and operate at partial load or is it better to choose the number of $C$-cores so that the torque required is the torque at full load? These two cases are shown in table II.

TABLE II

DESIGN OPTIONS

\begin{tabular}{lccc}
\hline Case & $N_{c}$ & Torque $(\mathrm{Nm})$ & $\cos (\phi)$ \\
\hline $\mathrm{A}$ & 24 & 32 & 0.314 \\
$\mathrm{~B}$ & 15 & 32 & 0.448 \\
\hline
\end{tabular}

Case $\mathrm{A}$ in table II is the machine designed only to maximise torque ( $N_{c}=24 \mathrm{C}$-cores) and operates at partial load to produce $32 \mathrm{Nm}$. Case $\mathrm{B}$ is the machine designed for $32 \mathrm{Nm}$ torque at full load $\left(N_{c}=15 \mathrm{C}\right.$-cores $)$. The results show that designing to maximise torque and operate at partial 
load (case A) has a significantly lower power factor than case B.

The basic idea behind this effect is that near the point of maximum torque the curve of torque curve has a very flat slope. On the other hand, the slope of the power factor curve is steep. These two effects implicate that a small increase of torque produces a strong penalty in terms of power factor. Therefore, when designing a TFM it is important to consider both the torque and the power factor.

\section{CONCLUSION}

This paper shows that even though TFMs are intrinsically three-dimensional it is possible to study them with intuitive analytical methods achieving a reasonable accuracy.

The case study clearly illustrates that if the design of the TFM is done only maximising torque, then the power factor will tend to be low. Therefore, it is important to have a tradeoff between torque and power factor particularly because small improvements of torque can produce strong penalties in terms of power factor. Furthermore, it is shown that the low power factor is not due to leakage in the classical way but due to the nature of the electromagnetic interaction that takes place.

Understanding the relationship between torque and power factor is a key step to unlocking the full potential of TFMs. This paper analyses a particular single-sided TFM but the concepts are general and can be applied to other topologies of TFM.

\section{REFERENCES}

[1] M. Mueller and H. Polinder, Electrical Drives for Direct Drive Renewable Energy Systems, 1st Edition. Woodhead Publishing., 2013.

[2] D. je Bang, H. Polinder, G. Shrestha, and J. A. Ferreira, "Comparative design of radial and transverse flux pm generators for direct-drive wind turbines," in Electrical Machines, 2008. ICEM 2008. 18th International Conference on, pp. 1-6, Sept 2008.

[3] O. Keysan, M. Mueller, A. McDonald, N. Hodgins, and J. Shek, "Designing the c-gen lightweight direct drive generator for wave and tidal energy," Renewable Power Generation, IET, vol. 6, pp. 161-170, May 2012.

[4] M. R. Harris, G. H. Pajooman, and S. M. A. Sharkh, "The problem of power factor in vrpm (transverse-flux) machines," in Electrical Machines and Drives, 1997 Eighth International Conference on (Conf. Publ. No. 444), pp. 386-390, Sep 1997.

[5] J. F. Gieras, "Performance characteristics of a transverse flux generator," in IEEE International Conference on Electric Machines and Drives, 2005., pp. 1293-1299, May 2005.

[6] D. H. Kang and H. Weh, "3D-FEM Analysis Versus Test Results for Three Transversal Flux Linear Motor Topologies," Electric Power Components and Systems, vol. 36, pp. 345-358, Mar. 2008.

[7] G. Yang, D. Cheng, H. Zhang, and B. Kou, "Bidirectional crosslinking transverse flux permanent magnet synchronous motor," IEEE Transactions on Magnetics, vol. 49, pp. 1242-1248, March 2013.

[8] N. J. Baker, S. Cawthorne, E. Hodge, and E. Spooner, "3d modelling of the generator for openhydro's tidal energy system," in Power Electronics, Machines and Drives (PEMD 2014), 7th IET International Conference on, pp. 1-6, April 2014

[9] H. Zhang, B. Kou, W. Zhao, and G. Yang, "Design and analysis of a bidirectional cross-linking transverse flux permanent magnet synchronous motor," in Electromagnetic Launch Technology (EML), 2014 17th International Symposium on, pp. 1-6, July 2014.

[10] M. R. Harris and B. C. Mecrow, "Variable reluctance permanent magnet motors for high specific output," in Electrical Machines and Drives, 1993. Sixth International Conference on (Conf. Publ. No. 376), pp. 437-442, Sep 1993.
[11] M. R. Harris, G. H. Pajooman, and S. M. A. Sharkh, "Performance and design optimisation of electric motors with heteropolar surface magnets and homopolar windings," IEE Proceedings - Electric Power Applications, vol. 143, pp. 429-436, Nov 1996.

[12] W. J. Gibbs, Conformal Transformations in Electrical Engineering. Chapman and Hall, 1958.

[13] E. Freeman, "The calculation of harmonics, due to slotting, in the fluxdensity waveform of a dynamo-electric machine," Proceedings of the IEE - Part C: Monographs, vol. 109, pp. 581-588, September 1962.

[14] G. H. Pajooman, Performance assessment and design optimisation of VRPM (transverse flux) machines by finite element computation. $\mathrm{PhD}$ Thesis, University of Southampton, 1997.

[15] K. Y. Lu, E. Ritchie, P. O. Rasmussem, and P. Sandholdt, "Modeling and power factor analysis of a single phase surface mounted permanent magnet transverse flux machine," in Power Electronics and Drive Systems, 2003. PEDS 2003. The Fifth International Conference on, vol. 2, pp. 1609-1613 Vol.2, Nov 2003.

[16] K. Lu, P. O. Rasmussen, and E. Ritchie, "Design considerations of permanent magnet transverse flux machines," IEEE Transactions on Magnetics, vol. 47, pp. 2804-2807, Oct 2011.

[17] M. F. J. Kremers, J. J. H. Paulides, J. L. G. Janssen, and E. A. Lomonova, "Analytical flux linkage and emf calculation of a transverse flux machine," in Electrical Machines (ICEM), 2014 International Conference on, pp. 2668-2673, Sept 2014.

[18] M. F. J. Kremers, J. J. H. Paulides, and E. A. Lomonova, "Toward accurate design of a transverse flux machine using an analytical 3-d magnetic charge model," IEEE Transactions on Magnetics, vol. 51, pp. 1-4, Nov 2015.

[19] M. G. Say, The Performance and Design of Alternating Current Machines. Sir Isaac Pitman \& Sons Ltd., 1965.

[20] P. Kundur, Power System Stability and Control. McGraw-Hill Professional, 1993.

[21] A. E. Fitzgerald, C. J. Kingsley, and S. D. Umans, Electric Machinery, Sixth Edition. McGraw-Hill Education, 2003.

[22] J. R. Hendershot and T. J. E. Miller, Design of Brushless PermanentMagnet Machines. Motor Design Books LLC, 2010.

[23] D. Zarko, D. Ban, and T. A. Lipo, "Analytical calculation of magnetic field distribution in the slotted air gap of a surface permanent-magnet motor using complex relative air-gap permeance," IEEE Transactions on Magnetics, vol. 42, pp. 1828-1837, July 2006.

[24] T. A. Driscoll, Schwarz-Christoffel Toolbox User's Guide: Version 2.3. Newark, DE: Dept. Math. Sci., Univ. Delaware, 2005.

\section{BIOGRAPHIES}

Jaime Renedo Anglada was born in Madrid, Spain in 1989. He received the M.Sc. degree in electrical engineering from the Comillas Pontifical University, Madrid, Spain, in 2013. He is currently pursuing the Ph.D. degree with the Electro-Mechanical Engineering Research Group at the University of Southampton, U.K. His main research topic is the analytic study of electric machines and drives.

From 2012 to 2013 he was an invited student at the Institute of Research in Technology (IIT), a research center of ICAI School of Engineering, which belongs to the Comillas Pontifical University, Madrid, Spain. The research topic was the modelling of synchronous generators for stability analysis.

Suleiman M. Sharkh received the B.Eng. and Ph.D. degrees in electrical engineering from the University of Southampton, Southampton, U.K., in 1990 and 1994, respectively.

He is Professor of Power Electronics, Machines and Drives, and head of the Electro-Mechanical Research Group at the University of Southampton. $\mathrm{He}$ is also the Managing Director of HiT Systems Ltd. He has published over 40 papers in academic journals and conferences. His main research interests are in the area of control, electrical machine and power electronics with applications to electric vehicles.

Prof. Sharkh is a member of the IEEE, the IET and a Chartered Engineer. He was the 2008 winner of The Engineer Energy Innovation Award for his work on rim driven thrusters and marine turbine generators. 